\title{
Ultraviolet and visual flux and line variations of one of the least variable Bp stars HD 64740^
}

\author{
J. Krtička ${ }^{1}$, J. Janík ${ }^{1}$, H. Marková ${ }^{1}$, Z. Mikulášek ${ }^{1,2}$, J. Zverko $^{1}$, M. Prvák ${ }^{1}$, and M. Skarka ${ }^{1}$ \\ 1 Department of Theoretical Physics and Astrophysics, Masaryk University, Kotlářská 2, 61137 Brno, Czech Republic \\ e-mail: krticka@physics.muni.cz \\ 2 Observatory and Planetarium of J. Palisa, VŠB - Technical University, 70833 Ostrava, Czech Republic
}

Received 29 December 2012 / Accepted 23 May 2013

\section{ABSTRACT}

\begin{abstract}
Context. The light variability of hot magnetic chemically peculiar stars is typically caused by the flux redistribution in spots with peculiar abundance. This raises the question why some stars with surface abundance spots show significant rotational light variability, while others do not.

Aims. We study the Bp star HD 64740 to investigate how its remarkable inhomogeneities in the surface distribution of helium and silicon, and the corresponding strong variability of many spectral lines, can result in one of the faintest photometric variabilities among the Bp stars.

Methods. We used model atmospheres and synthetic spectra calculated for the silicon and helium abundances from surface abundance maps to predict the ultraviolet and visual light and line variability of HD 64740. The predicted fluxes and line profiles were compared with the observed ones derived with the IUE, HST, and HIPPARCOS satellites and with spectra acquired using the FEROS spectrograph at the $2.2 \mathrm{~m}$ MPG/ESO telescope in La Silla.

Results. We are able to reproduce the observed visual light curve of HD 64740 assuming an inhomogeneous distribution of iron correlated with silicon distribution. The light variations in the ultraviolet are hardly detectable. We detect the variability of many ultraviolet lines of carbon, silicon, and aluminium and discuss the origin of these lines and the nature of their variations.

Conclusions. The maximum abundances of helium and silicon on the surface of HD 64740 are not high enough to cause significant light variations. The detected variability of many ultraviolet lines is most likely of atmospheric origin and reflects the inhomogeneous elemental surface distribution. The variability of the CIV resonance lines of carbon is stronger and it probably results from the dependence of the wind mass-loss rate on the chemical composition and magnetic field orientation. We have not been able to detect a clear signature of the matter trapped in the circumstellar clouds.
\end{abstract}

Key words. stars: chemically peculiar - stars: early-type - stars: variables: general - stars: winds, outflows - ultraviolet: stars stars: individual: HD 64740

\section{Introduction}

Atmospheres of stars from the upper part of the main sequence provide us with a unique laboratory for studying the fascinating effects of the radiative force. While in the hot luminous stars the radiative force is strong enough to launch a line-driven wind (Puls et al. 2008), the atmospheres of numerous less luminous stars are relatively quiet. Consequently, the diffusion under the influence of the radiative and gravitational forces causes pronounced deviations from the typical chemical composition (Vauclair 2003; Michaud 2004). Stars with such atmospheres are called chemically peculiar.

Chemically peculiar (CP) stars constitute a very diverse group of stars, and the deviations from the solar chemical composition is the only attribute common to all of them. The stars may differ in the strength of their peculiarity (Briquet et al. 2004), elements with peculiar abundance (Romanyuk 2007), horizontal and vertical homogeneity (Ryabchikova et al. 2008), variability (Mikulášek et al. 2007), stability of their rotational periods (Mikulášek et al. 2011), and the presence and strength of the magnetic field (Landstreet et al. 2008), to mention the most important differences.

^ Partly based on observations obtained at the European Southern Observatory (ESO programme 089.D-0153(A)).
Helium-rich stars are among the most enigmatic objects of the group of $\mathrm{CP}$ stars. In the Hertzsprung-Russell diagram they lie at the border between stars with strong line-driven winds and stars with weak winds, which can be hardly detected. The hottest ones have winds with typical mass-loss rates of about $10^{-9} M_{\odot}$ year $^{-1}$. The wind can fill stellar magnetospheres in the presence of the magnetic field, creating magnetospheric clouds (Landstreet \& Borra 1978; Shore \& Brown 1990; Smith \& Groote 2001; Townsend et al. 2005).

Light, and consequently also the spectral energy distribution variability, is a typical property of many $\mathrm{CP}$ stars. There are several types of light variability observed in CP stars, including pulsations (Balona et al. 2011; Paunzen et al. 2013), or the variability resulting from the light absorption in magnetospheric clouds (Townsend et al. 2005). However, the most typical light variability observed in $\mathrm{CP}$ stars is the rotational one, which is connected with the presence of spots on the surface of $\mathrm{CP}$ stars. The flux redistribution due to the bound-bound (line) and boundfree (continuum) transitions in the inhomogeneously distributed chemical elements on the surface of a rotating star (Peterson 1970; Molnar 1973; Lanz et al. 1996; Lüftinger et al. 2010) results in the rotational variability of a CP star. The typical amplitude of the rotational variability is on the order of hundredths of magnitude. 
Precise abundance maps (Kochukhov et al. 2012) enable us to successfully simulate the light variability of CP stars. Several main sources of the rotational light variability of $\mathrm{CP}$ stars were identified. Bound-free transitions of silicon and line transitions of iron seem to be the most universal ones (Krivosheina et al. 1980; Krtička et al. 2009) operating in CP stars with different effective temperatures. Other elements may contribute to the light variability in specific spectral types, including helium in hot helium-rich stars (Krtička et al. 2007), and chromium in cooler CP stars (Shulyak et al. 2010a; Krtička et al. 2012).

It is interesting to investgate, why some stars display strong light variations and others do not. The helium-rich star HD 64740 (HR 3089, Hiltner et al. 1969) belongs to the latter ones, and was marked by Adelman (2001) as one of the least variable stars in HIPPARCos photometry. While this designation may seem outdated in the era of space-based photometry with micromagnitude precision (Balona 2011), we feel that it is still meaningful in comparison with stars with much higher light variability amplitudes. Similarly to other helium-rich stars, HD 64740 has a strong magnetic field (Borra \& Landstreet 1979) and shows emission of circumstellar origin in the wings of $\mathrm{H} \alpha$ (Alecian et al. 2012). The star is an X-ray source (Drake et al. 1994) located in an interesting part of the sky at the borders of the well-known Gum nebula (Edgar \& Savage 1992).

The abundance of chemical elements in the spots on HD 64740 surface was derived by Bohlender (1988, hereafter B88, see also Bohlender \& Landstreet 1990). Clearly, the star HD 64740 is an ideal target for studying the light and spectral energy distribution variability of least-variable CP stars.

\section{Observations}

When studying periodically variable observables, such as light curve of a star, it is important to know the period and ephemeris of the variability. We attempted to derive a new ephemeris for the studied star using the observations available to us, i.e., the historical photometry, and optical and ultraviolet spectroscopy. However, we were not successful mainly because of the lack of suitable information about the phase in the data available to us. The photometric data are very noisy, the UV spectroscopy covers only a limited time interval and the optical spectroscopy is incomplete. Consequently, we adopted the ephemeris derived by Bohlender et al. (1987)

$\mathrm{JD}=(2444611.859 \pm 0.042)+(1.33026 \pm 0.00006) E$

to calculate the rotational phase. The zero-phase of this ephemeris is centered on the minimum of the magnetic field. The ephemeris is fairly precise, but the precision is not high enough to accommodate modern data as well. This is not a problem for the key data on which this study is based (IUE and HIPPARCOS observations), because they were obtained in the years 1981-1992, where the uncertainty of the phase is only about 0.1 . On the other hand, the uncertainty of the phase of the data derived after 2005 exceeds 0.3 , consequently, these data cannot be used for any reliable phase-dependent analysis.

\subsection{UV spectroscopy}

We used IUE observations of HD 64740 extracted from the INES database (Wamsteker et al. 2000, see Table 1) using the SPLAT package (Draper 2004; see also Škoda 2008). We used highdispersion large aperture spectra in the domains 1150-1900 $\AA$ (SWP camera) and 2000-3200 A (LWP camera). The relatively
Table 1. List of IUE and HST observations of HD 64740 used here.

\begin{tabular}{lllcl}
\hline \hline Satellite & Camera & Image & $\begin{array}{c}\text { Julian date } \\
2400000+\end{array}$ & Phase \\
\hline IUE & SWP & 14442 & 44796.21075 & 0.583 \\
IUE & SWP & 14459 & 44798.18414 & 0.067 \\
IUE & SWP & 14472 & 44800.17412 & 0.563 \\
IUE & SWP & 14491 & 44802.17227 & 0.065 \\
IUE & SWP & 14508 & 44804.19079 & 0.582 \\
IUE & SWP & 14659 & 44822.04922 & 0.007 \\
IUE & SWP & 14686 & 44824.24160 & 0.655 \\
IUE & SWP & 15760 & 44953.89902 & 0.123 \\
IUE & SWP & 15809 & 44958.62916 & 0.678 \\
IUE & SWP & 19101 & 45362.52972 & 0.304 \\
IUE & SWP & 19104 & 45362.63918 & 0.386 \\
IUE & SWP & 19106 & 45362.70596 & 0.436 \\
IUE & SWP & 19108 & 45362.77153 & 0.486 \\
IUE & SWP & 19118 & 45363.65535 & 0.150 \\
IUE & SWP & 19121 & 45363.75405 & 0.224 \\
IUE & SWP & 19123 & 45363.81854 & 0.273 \\
IUE & SWP & 19132 & 45364.57750 & 0.843 \\
IUE & SWP & 19135 & 45364.70901 & 0.942 \\
IUE & SWP & 19138 & 45364.80958 & 0.018 \\
IUE & SWP & 19146 & 45365.55573 & 0.579 \\
IUE & SWP & 19149 & 45365.66782 & 0.663 \\
IUE & SWP & 19151 & 45365.73433 & 0.713 \\
IUE & SWP & 19153 & 45365.81644 & 0.774 \\
IUE & LWP & 12335 & 47150.62037 & 0.470 \\
HST & STIS & & 55319.46898 & \\
\hline & & & &
\end{tabular}

high number of SWP spectra allowed us to study the ultraviolet (UV) light variability of the star, but only information about the flux distribution could be obtained from the sole LWP spectrum. The SWP spectra are available in two spectral resolutions. In most applications we used spectra with the higher spectral resolution of $0.2 \AA$. The uncertainty of the phase of IUE/SWP observations is $0.04-0.05$ from the ephemeris Eq. (1), consequently, the phase is known precisely for these data. The uncertainity of the phase of IUE/LWP observation is somewhat larger, about 0.1 . There are also six narrow Hubble Space Telescope (HST)/STIS spectra available. All these spectra were obtained nearly in the same phase, therefore we selected only one of them for our study. The phase of HST/STIS observations has a too large uncertainty of 0.4 (from Eq. (1)), hence it does not bear any information and we did not include it in Table 1 . There is also a Copernicus spectrum available for HD 64740 (Lester 1976), but its wavelength region mostly coincides with the IUE one, so we did not use it here.

In Fig. 1 we compare the mean observed UV flux with the minimum and maximum one at each wavelength. Moreover, we used two different tests to detect the line variability. We plot the dispersion of the data divided by a mean flux at each wavelength, $\sigma\left(F_{\lambda}\right) / \bar{F}_{\lambda}$, and the amplitude of the flux variability

$A_{\lambda}=\sqrt{8 \int_{0}^{1} \Delta F_{\lambda}^{2}(\varphi) \mathrm{d} \varphi}$,

where the difference between the fit by a simple polynomial $F_{\lambda}(\varphi)$ (with parameters $\bar{F}_{\lambda}, a_{\lambda}$, and $b_{\lambda}$ ) and the mean flux at a given phase $\varphi$ is

$\Delta F_{\lambda}(\varphi)=F_{\lambda}(\varphi)-\bar{F}_{\lambda}=a_{\lambda} \cos (2 \pi \varphi)+b_{\lambda} \sin (2 \pi \varphi)$.

The coefficients $a_{\lambda}$ and $b_{\lambda}$ are fitted for each wavelength of the observed spectra. This gives $\Delta F_{\lambda}(\varphi)$, which is a continuous 
J. Krtička et al.: Modelling ultraviolet and visual spectral energy distribution variations of Bp star HD 64740

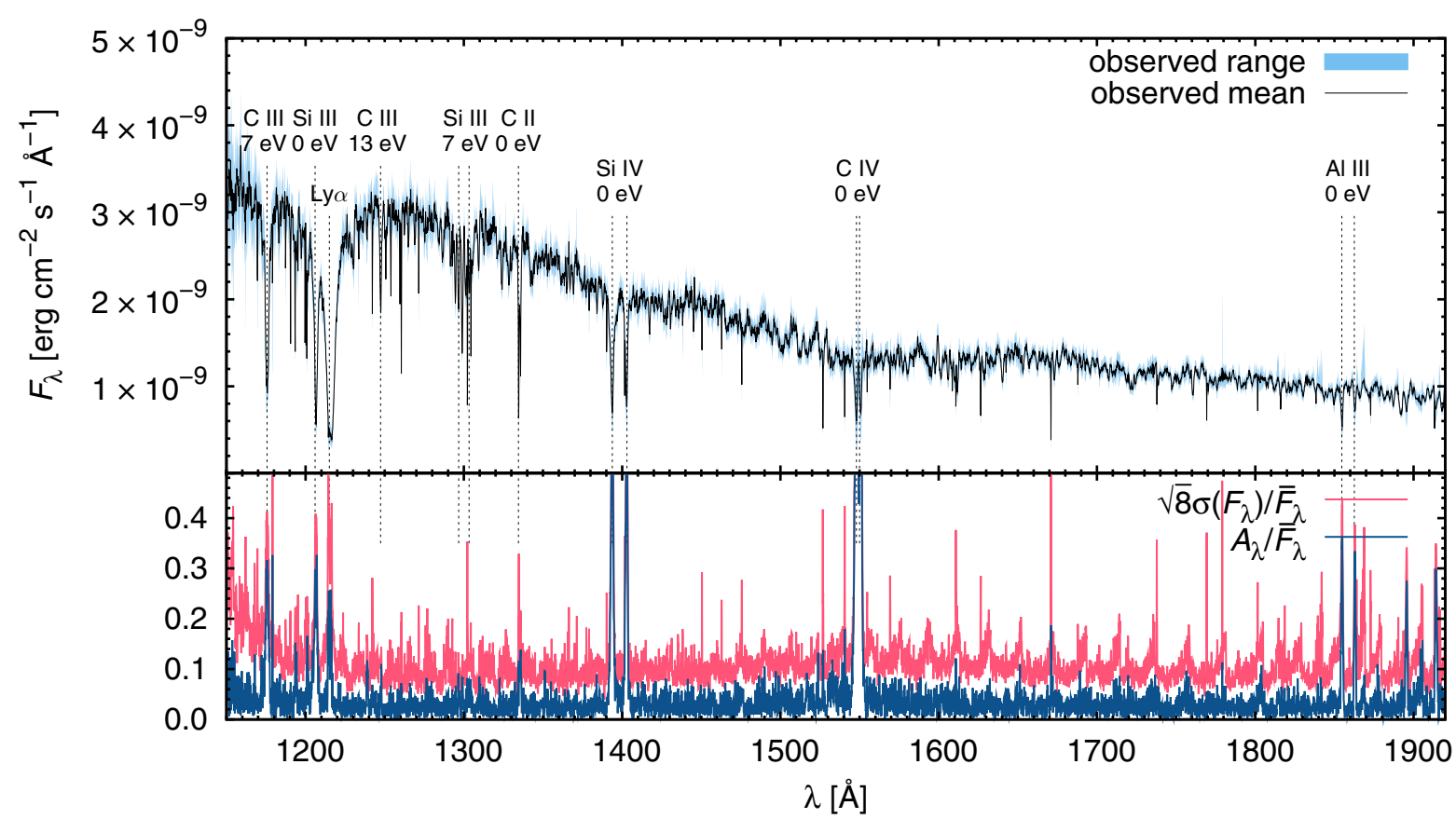

Fig. 1. Observed far-ultraviolet fluxes of HD 64740 and their variations. The fluxes were smoothed with a Gaussian filter with a dispersion of $0.1 \AA$ to reduce the noise. Upper panel: mean flux (solid line) and the range of flux variations (lighter color). Lower panel: the wavelength dependence of the flux dispersion (over the rotational period) and the effective amplitude Eq. (2) divided by the mean flux.

function of $\varphi$ and can be integrated in Eq. (2). With this fit we should be able to detect any variability with a nonzero sinusoidal component.

A normalization of the dispersion by the mean flux $\sigma\left(F_{\lambda}\right) / \bar{F}_{\lambda}$ in Fig. 1 may lead to artificial peaks at the line positions even if the line is not variable. For a sinusoidal signal in the limit of large uniformly distributed dataset the dispersion is connected with amplitude as $A_{\lambda}=\sqrt{8} \sigma\left(F_{\lambda}\right)$. This can be used to infer the reliability of the peaks in Fig. 1, but in general a visual inspection of the data (or advanced statistical techniques) is necessary to test the significance of individual peaks. From Fig. 1 the flux variability can be detected in the strongest lines, which are mostly resonance lines, whereas weaker lines show no strong variability. The presence of resonance lines is an argument for the existence of the magnetically confined circumstellar environment around HD 64740 where only ground levels of ions are strongly populated, and their variability suggests that the environment is co-rotating (Shore \& Brown 1990). The varying lines originating from excited levels also indicate atmospheric variability. The amplitude of the variations of pseudo-continuum, which is mostly formed by numerous absorption lines, varies with wavelength. This points to another process that contributes to the pseudo-continuum variability in addition to the observing noise. Higher variability in the region of $1500-1600 \AA$ indicates an inhomogeneous surface distribution of iron (see Sect. 4).

We were unable to detect any significant rotationally modulated broadband flux variation (see also Fig. 5) except for the variations at $1550 \AA$. The variations at $1550 \AA$ are caused by variable $\mathrm{C}$ IV lines and possibly also by numerous iron lines, indicating an inhomogeneous flux distribution of iron (see Sect. 4).

\subsection{Optical spectroscopy}

During run 089.D-0153(A) at the La Silla Observatory (2.2 m MPG/ESO telescope) we obtained new 12 FEROS echelle spectra with a resolution $R=48000$. Initial reductions of the
Table 2. List of new FEROS echelle spectra used here.

\begin{tabular}{cccc}
\hline \hline Telescope & $\begin{array}{c}\text { Julian date } \\
2400000+\end{array}$ & Phase & $\begin{array}{c}\text { Exposure time } \\
{[\mathrm{s}]}\end{array}$ \\
\hline MPG/ESO & 56157.88494 & 0.526 & 180 \\
MPG/ESO & 56157.89988 & 0.537 & 180 \\
MPG/ESO & 56157.91512 & 0.548 & 180 \\
MPG/ESO & 56157.93037 & 0.560 & 180 \\
MPG/ESO & 56158.88036 & 0.274 & 180 \\
MPG/ESO & 56158.90260 & 0.291 & 180 \\
MPG/ESO & 56158.91762 & 0.302 & 180 \\
MPG/ESO & 56158.93276 & 0.313 & 180 \\
MPG/ESO & 56159.87578 & 0.022 & 240 \\
MPG/ESO & 56159.89144 & 0.034 & 240 \\
MPG/ESO & 56159.90722 & 0.046 & 240 \\
MPG/ESO & 56159.92286 & 0.058 & 240 \\
\hline
\end{tabular}

spectra and their conversion into 1D images were carried out by JJ, who used IRAF (bias-subtraction and flat-field calibration). The wavelength calibrations were based on ThAr-Ne comparison spectra. The wavelength coverage of these spectra is 3730-7900 $\AA$. The journal of new spectra on which this study is based is presented in Table 2. The phase of individual spectroscopic observations has according to Eq. (1) a too large uncertainty of 0.4 , therefore we used the phase only to label individual spectra in Table 2.

\subsection{Photometry}

The visual light variations are hard to detect (Adelman 2001, see also Fig. 5). The amplitude of the light variability in the HIPPARCOS $H_{\mathrm{p}}$ filter (ESA 1997) is only about $0.004 \mathrm{mag}$. The minimum of the light variations observed around the zero phase coincides with the maximum flux at $1550 \AA$. This indicates that the inhomogeneous surface distribution of iron could be one of the causes of the light variability. This is also supported 
Table 3. List of new $U B V$ observations used here.

\begin{tabular}{cccl}
\hline \hline Date & $\begin{array}{c}\text { Epoch } \\
2455990+\end{array}$ & $\begin{array}{c}\text { No. of } \\
\text { obs. }\end{array}$ & Comparison \\
\hline 9.3 .2012 & $6.2872-6.4615$ & 36 & all sky \\
10.3 .2012 & $7.2647-7.4461$ & 46 & all sky \\
11.3 .2012 & $8.2645-8.4618$ & 42 & all sky \\
12.3 .2012 & $9.2590-9.4442$ & 42 & all sky \\
\hline
\end{tabular}

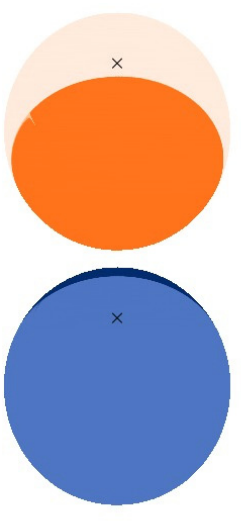

$\varphi=0.000$

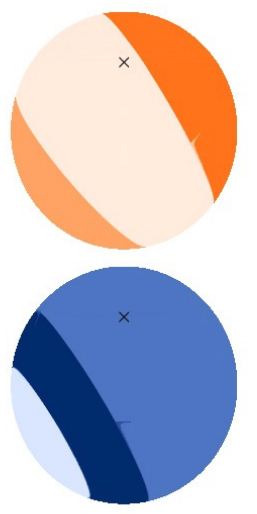

$\varphi=0.333$
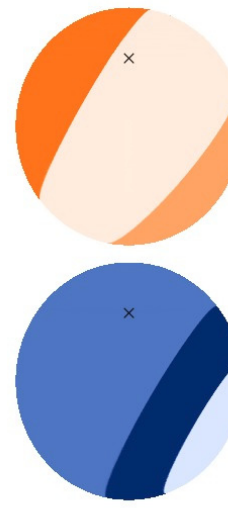

$\varphi=0.667$
Fig. 2. Distribution of helium (upper panel) and silicon (lower panel) on the surface of HD 64740 in three different phases.

Table 4. Adopted HD 64740 parameters (B88).

\begin{tabular}{lc}
\hline \hline Effective temperature $T_{\text {eff }}$ & $24000 \pm 1000 \mathrm{~K}$ \\
Surface gravity $\log g(\mathrm{cgs})$ & $4.0 \pm 0.15$ \\
Inclination $i$ & $36^{\circ} \pm 15^{\circ}$ \\
Projection of the rotational velocity $v \sin i$ & $140 \pm 10 \mathrm{~km} \mathrm{~s}^{-1}$ \\
Angle between rotation and magnetic axis $\beta$ & $78^{\circ} \pm 8^{\circ}$ \\
Stellar mass $M$ & $11.5 \pm 2 M_{\odot}$ \\
Stellar radius $R_{*}$ & $6.3 \pm 1.8 R_{\odot}$ \\
Polar magnetic field strength $B_{*}$ & $3700 \mathrm{G}$ \\
\hline
\end{tabular}

by the observations made by Pedersen \& Thomsen (1977) in Strömgren $y$, that show similar light variability.

Two of us (JJ and MS) obtained 166 new $U B V$ photometric observations during four photometric nights (9.-12.3.2012) using the 20" telescope in South Africa Observatory with modular photometer. All these observations were obtained using allsky as well as differential (relative to HD 63578) photometry. Observations were reduced with the help of the reduction program HEC22 (rel. 14), which uses non-linear formulæ to transform from the natural to the standard $U B V$ system (Harmanec et al. 1994; Harmanec \& Horn 1998). The journal of new photoelectric observations on which this study is based is presented in Table 3.

\section{Method of calculation}

\subsection{Stellar parameters}

In Table 4 we list the stellar parameters and their uncertainties estimated by B88, who used photometry for the effective temperature, and fitting of hydrogen and helium lines for the surface gravity. We used the four complete sets of $u v b y \beta$ indexes listed in the SIMBAD database and calculated the effective temperature applying the codes UVBYBETA (Moon \& Dworetsky 1985) and TEFFLOG (Smalley \& Dworetsky 1995). The former
Table 5. Parameters of two helium spots and silicon belts.

\begin{tabular}{ccccc}
\hline \hline Helium & Colatitude & Longitude & Radius & $\varepsilon_{\mathrm{He}}$ \\
& $0^{\circ}$ & $0^{\circ}$ & $70^{\circ}$ & -0.456 \\
& $160^{\circ}$ & $180^{\circ}$ & $60^{\circ}$ & -0.523 \\
& \multicolumn{2}{c}{ outside the spots } & & -1.0 \\
\hline Silicon & \multicolumn{2}{c}{ Colatitude } & $\varepsilon_{\mathrm{Si}}$ & $\varepsilon_{\mathrm{Fe}}$ \\
& $0^{\circ}-110^{\circ}$ & -4.3 & -4.4 \\
& $110^{\circ}-140^{\circ}$ & -3.6 & -3.8 \\
& $140^{\circ}-180^{\circ}$ & -6.0 & -5.9 \\
\hline
\end{tabular}

Notes. Helium and silicon abundances were derived by B88, while $\varepsilon_{\mathrm{Fe}}$ is the iron abundance assumed here.

resulted in a $T_{\text {eff }}$ from 24300 to $25000 \mathrm{~K}$, while the latter gives values from 23100 to $23700 \mathrm{~K}$ with a formal error $\pm 800 \mathrm{~K}$. The difference between the extreme values reaches nearly $2000 \mathrm{~K}$, which suggests a large uncertainity in the determination of the effective temperature, and its influence should be taken into account. Consciuos of this, we considered it reasonable to adopt the set of parameters from the BSTAR2006 (Lanz \& Hubeny 2007) grid, which are very similar to that for which the abundance maps were calculated. The projected rotational velocity was derived by B88 from helium lines.

The parameters of spots and the final adopted abundances in different regions on the HD 64740 surface are given in Table 5 (see also Fig. 2). Here the abundances are given as the logarithm of the elemental number density $N_{\mathrm{el}}$ relative to the number density of all elements $N_{\text {tot }}, \varepsilon_{\mathrm{el}}=\log \left(N_{\mathrm{el}} / N_{\mathrm{tot}}\right)$. The coordinates in Table 5 are given with respect to the magnetic pole. There are two spots with enhanced helium abundance located nearly opposite to each other with respect to the star's center and three belts with a different silicon abundance. There is also a nitrogen spot on the surface of HD 64740 (B88), but our test showed that as a result of its relatively low abundance, nitrogen does not significantly contribute to the light curve. Consequently, we assumed $\varepsilon_{\mathrm{N}}=-4.7$, corresponding to the nitrogen abundance outside the spots. The abundance of iron was selected to match the visual light curve, see Sect. 5.

The stellar surface was divided in to $90 \times 45$ surface elements in longitude and latitude. We calculated model atmospheres and synthetic spectra for the chemical composition found in each surface element.

\subsection{Model atmospheres and synthetic spectra}

We used the code TLUSTY to calculate the NLTE plane-parallel model atmospheres (Lanz \& Hubeny 2003). The atomic data to calculate the model atmospheres are the same as those used by Lanz \& Hubeny (2007).

To calculate the model atmospheres and synthetic spectra we assumed fixed values of the effective temperature and surface gravity (according to Table 4) and adopted a generic value of the microturbulent velocity $v_{\text {turb }}=2 \mathrm{~km} \mathrm{~s}^{-1}$. The abundance of helium, silicon, and iron corresponds to the abundance found in individual surface regions (see Table 5). We used the solar abundance of other elements (Asplund et al. 2005, except nitrogen, see Sect. 3.1). We updated the oscillator strengths of the strong silicon lines in our line list using the data from the Atomic Line List $^{1}$.

To predict the spectral energy distribution variations, we computed specific intensities $I\left(\lambda, \theta, \varepsilon_{\mathrm{He}}, \varepsilon_{\mathrm{Si}}\right)$ for 20 equidistantly

1 http://www.pa.uky.edu/ peter/atomic/ 
J. Krtička et al.: Modelling ultraviolet and visual spectral energy distribution variations of Bp star HD 64740

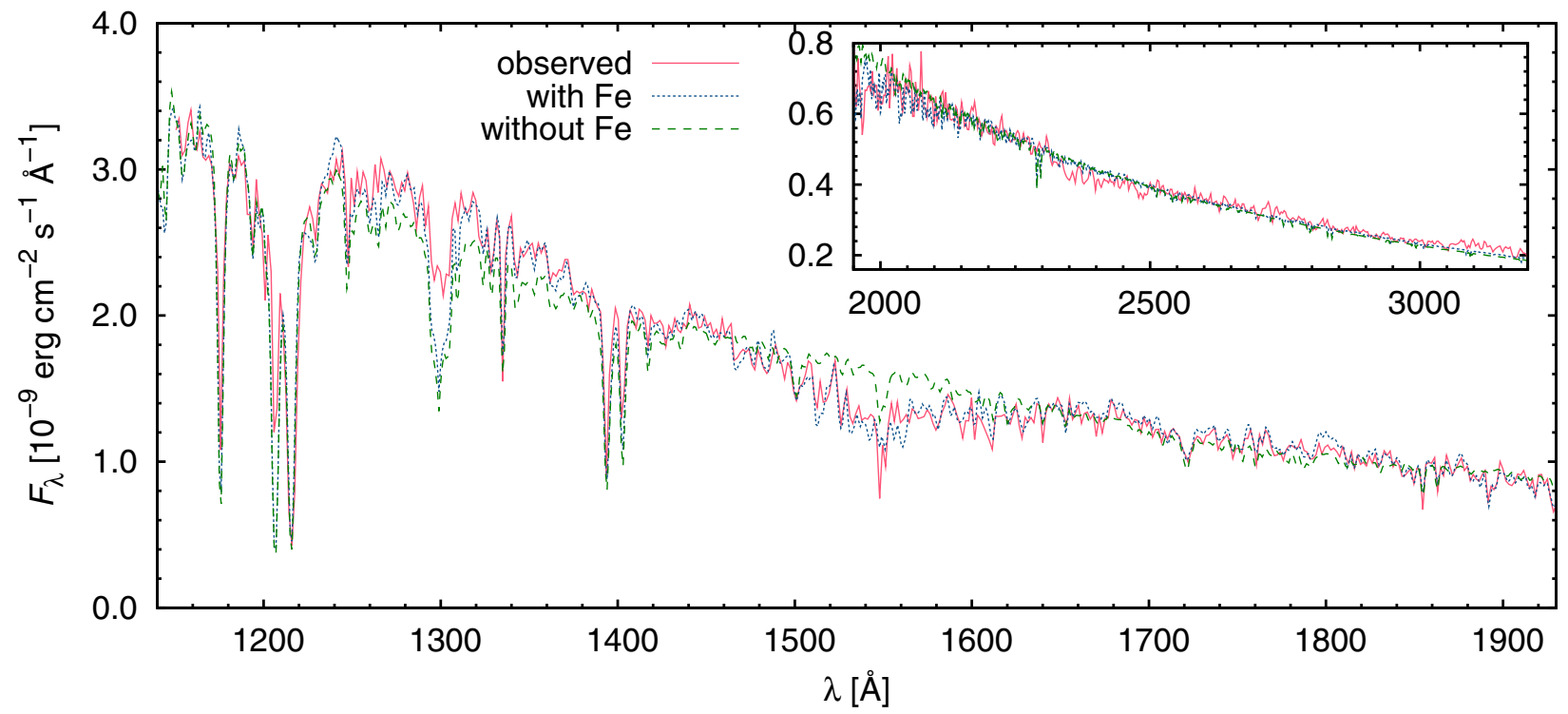

Fig. 3. Comparison of observed UV flux (IUE, mean low spectral resolution spectrum) with flux from model atmospheres for solar iron abundance $\varepsilon_{\mathrm{Fe}}=-4.6$ and for a model without iron (the abundance of helium and silicon is $\varepsilon_{\mathrm{He}}=-1.0, \varepsilon_{\mathrm{Si}}=-4.3$ ). The depression at $1550 \AA$ is a clear signature of iron. Both model fluxes were multiplied by a constant factor to match the observed spectrum and convolved with a Gaussian filter with a dispersion of $1.3 \AA$. The LWP12335 spectrum and predicted near-UV fluxes are overplotted in the upper right.

spaced values of $\mu=\cos \theta$, where $\theta$ is the angle between the normal to the surface and the line of sight. The intensities were calculated by the SYNSPEC code assuming NLTE from the TLUSTY model atmospheres. We also took into account the same transitions as for the model atmosphere calculations (see Krtička et al. 2012). Additionally, we included the lines of all elements with the atomic number $Z \leq 30$ that were not accounted in the model atmosphere calculation.

\subsection{Phase-dependent flux distribution}

The radiative flux in a color $c$ at the distance $D$ from the star with radius $R_{*}$ is (Mihalas 1978)

$$
f_{\mathrm{c}}=\left(\frac{R_{*}}{D}\right)^{2} \int_{\substack{\text { visible } \\ \text { surface }}} I_{\mathrm{c}}(\theta, \Omega) \cos \theta \mathrm{d} \Omega .
$$

The integral Eq. (4) is calculated numerically over all visible surface elements taking into account the star's rotation. The intensity $I_{\mathrm{c}}(\theta, \Omega)$ at each surface point with spherical coordinates $\Omega$ is an integral over the specific intensity for the appropriate abundance of helium, silicon, and iron,

$$
I_{\mathrm{c}}(\theta, \Omega)=\int_{0}^{\infty} \Phi_{\mathrm{c}}(\lambda) I\left(\lambda, \theta, \varepsilon_{\mathrm{He}}, \varepsilon_{\mathrm{Si}}\right) \mathrm{d} \lambda .
$$

The response function $\Phi_{c}(\lambda)$ of a given filter $c$ is approximated by a polynomial fit of tabulated values.

The magnitude difference is defined as

$\Delta m_{\mathrm{c}}=-2.5 \log \left(\frac{f_{\mathrm{c}}}{f_{\mathrm{c}}^{\text {ref }}}\right)$,

where $f_{\mathrm{c}}$ is calculated from Eq. 4 for a given rotational phase and $f_{\mathrm{c}}^{\text {ref }}$ is the reference flux obtained under the condition that the mean magnitude difference over the rotational period is zero.

\section{Mean UV fluxes and the $1550 \AA$ iron depression}

Iron lines substantially determine the stars' ultraviolet flux distribution (cf., Shulyak et al. 2010b). This is documented in Fig. 3, where we plot the UV fluxes computed for the solar iron abundance, for the iron-free atmosphere, and the observation. The predicted fluxes were scaled by a constant factor to derive the best match between theory and observation. Both models with and without iron agree with the observed spectrum for longer UV wavelengths $\lambda \gtrsim 1600 \AA$. However, in the region of $1500-1600 \AA$ the flux calculated without iron is significantly higher than the observed one, while it is somewhat lower in the region of $1250-1400 \AA$. The flux with solar iron abundance agrees well with observations in the far-UV region (1150-1900 $⿱$ ) , both in general shape and in many details (except for some stronger lines, e.g., Si III lines at $1206 \AA$ and $1300 \AA$ ). The connection between the $1550 \AA$ feature and iron was previously shown by Brown et al. (1985). The flux variability at these wavelengths is a proxy for the inhomogeneous surface distribution of iron (cf., Sokolov 2006).

Comparison of predicted near UV fluxes (2000-3200 $⿱$ ) and one available near UV observation in Fig. 3 shows that the flux distribution is well reproduced, however most of minute features in the observed spectrum are not present in the predicted spectrum. It is not clear what is the cause of this difference. Either some line opacity is still missing in this region due to the peculiar elemental abundances that we assume to be the solar ones, or the observed flux is less certain here.

\section{Predicted light variations}

Predicted light curves were calculated from the surface abundance maps derived by B88 (see also Table 5) and from the emergent fluxes computed with the SYNSPEC code, applying Eq. (6) for individual rotational phases.

Strong far-UV opacity due to helium and silicon boundfree transitions causes the redistribution of the flux from the far-UV region to the near-UV and visible ones. Consequently, 


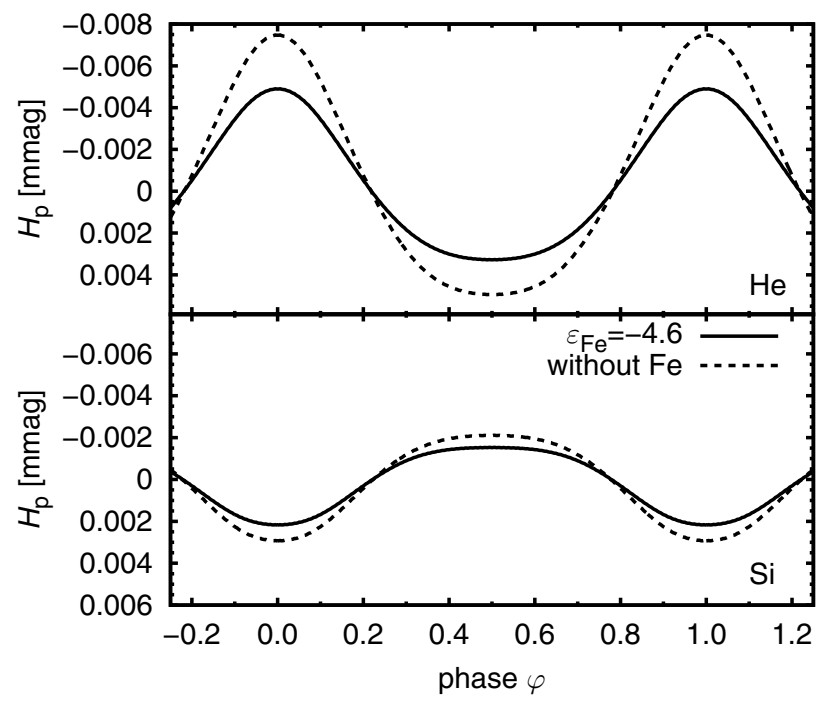

Fig. 4. Predicted light variations of HD 64740 in $H$ p photometric filter calculated using abundance maps of one element only. The abundance of other elements was fixed. Upper plot: light variations due to helium only. Silicon abundance was fixed to $\varepsilon_{\mathrm{Si}}=-4.3$. Lower plot: light variations due to silicon only. The helium abundance was fixed to $\varepsilon_{\mathrm{He}}=-1.0$. Light curves were calculated using the solar iron abundance $\left(\varepsilon_{\mathrm{Fe}}=-4.6\right.$, solid lines) and without iron (dashed lines).

surface spots with enhanced helium and silicon abundance are bright in the visible and dark in the far-UV regions (Krtička et al. 2007). The visual light maximum is therefore observed at the phase of the strongest lines of either helium or silicon. This is demonstrated in Fig. 4, where we plot the light variations calculated assuming the inhomogeneous surface distribution of one element only, while the other element is distributed uniformly over the whole surface. The light variations due to helium and silicon are in antiphase, because helium-overabundant regions are seen on the stellar surface mostly around the phase $\varphi=$ 0.0 , whereas regions with enhanced silicon abundance are seen mostly around the phase $\varphi=0.5$. Note also that one of the helium spots never dominates in the region that directly faces the observer (see Fig. 2), hence there is only one wave in the helium light curve.

From Fig. 4 it follows that iron influences the light curve even if it is homogeneously distributed on the stellar surface. The reason for this is that iron reduces the relative opacity variations and consequently also the flux variations caused by other elements, therefore the iron-free atmosphere displays a larger variability amplitude than the atmosphere with a normal, solar iron abundance distributed uniformly over the surface.

Taking into account the surface distribution of helium and silicon, the predicted light curves disagree with observed ones. The observed light maximum occurs around the phase of the maximum strength of silicon lines, $\varphi=0.5$, but the predicted light curve is dominated by helium, peaking around the phase $\varphi=0.0$ (Fig. 5). This can be deduced from Fig. 4, where the amplitude of the light variations due to helium is significantly larger than that due to silicon. From this we conclude that there is another element that contributes to the light curve. From the ultraviolet observations available we assume that this missing element is iron.

We accounted for the assumed inhomogeneous iron surface distribution in the calculation of the light curves. Because the corresponding iron surface map is missing, we assumed that iron has the same spot structure as silicon. A rough correlation

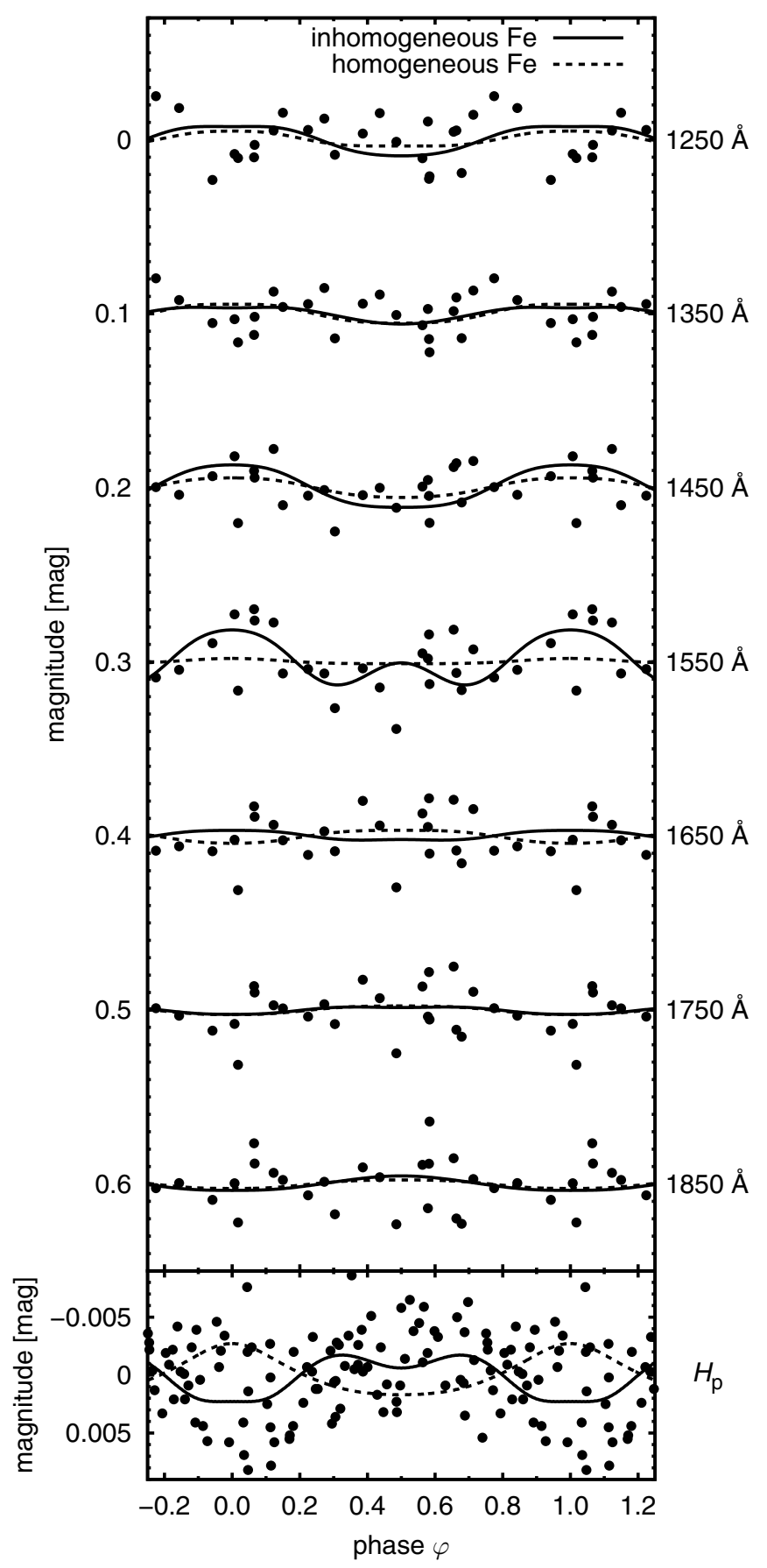

Fig. 5. Predicted light variations of HD 64740 computed taking into account the helium and silicon surface abundance distributions after Table 5 (solid lines: inhomogeneous iron distribution after Table 5, dashed lines: homogeneous iron distribution) compared with IUE and HIPPARCOS observations (dots). Upper panel: the far ultraviolet region. Observed and predicted fluxes were smoothed by a Gaussian filter as described in the text. Curves for individual wavelengths were vertically shifted. The region around CIV $\lambda 1548$ and $\lambda 1551$ lines was excluded from the analysis. Bottom panel: the optical region in the HIPPARCos $H_{\mathrm{p}}$ filter.

between the iron and silicon abundance is typical in many CP stars (e.g., Lehmann et al. 2007). We tried several different combinations of iron abundance to match the observed light curve. The best agreement was obtained assuming that the iron abundance scales as $\varepsilon_{\mathrm{Fe}}=\varepsilon_{\mathrm{Fe}, \odot}\left(\varepsilon_{\mathrm{Si}} / \varepsilon_{\mathrm{Si}, \odot}\right)^{0.85}$, where $\odot$ denotes solar abundance of a given element. The iron abundances 
Table 6. Effective amplitude Eq. (7) of the light variations in individual optical bands derived from observed and predicted light curves.

\begin{tabular}{lcccc}
\hline \hline Band & $U$ & $B$ & $H_{\mathrm{p}}$ & $V$ \\
Observed $A_{\text {eff }}[\mathrm{mmag}]$ & $2.1 \pm 1.4$ & $5.2 \pm 1.3$ & $3.7 \pm 1.1$ & $4.1 \pm 1.3$ \\
Predicted $A_{\text {eff }}[\mathrm{mmag}]$ & 2.9 & 4.8 & 3.7 & 2.9 \\
\hline
\end{tabular}

calculated using this formula are given in Table 5. The predicted $H_{\mathrm{p}}$ light curve corresponds to the observed one and the predicted minute UV flux variations do not contradict the observed flux variations (Fig. 5). Here we applied a Gaussian function with $\sigma=25 \AA$ as the response function of the filter, centered at the corresponding wavelengths to obtain UV broadband flux variations after Eq. (4). The importance of iron is especially visible in the region of $1550 \AA$ and in the optical region.

The predicted minute light variations in other visual bands do not disagree with observations either. This can be seen from Table 6, where we compare the effective amplitude of the light variations $A_{\text {eff }}$ derived using observed and predicted data in individual bands. We compare only the effective amplitude and not the light curves, because the observations are relatively noisy and the uncertainty of the phase of the $U B V$ observations is large. The effective amplitude is defined here as

$A_{\text {eff }}=\sqrt{8 \int_{0}^{1} \Delta m^{2}(\varphi) \mathrm{d} \varphi}$

where $\Delta m(\varphi)$ the difference between the magnitude at a given phase $\varphi$ and the mean amplitude. For each band $\Delta m(\varphi)$ is derived from a simple polynomial fit

$\Delta m(\varphi)=a \cos (2 \pi \varphi)+b \sin (2 \pi \varphi)$,

where $a$ and $b$ are the parameters of the fit. We note that in the Balmer continuum (in the region of Strömgren $u$ ) even these minute changes nearly cancel, resulting in smaller amplitude in $U$ than in $B$.

\section{Variability of UV lines}

Figure 1 shows that many UV lines might be variable. The presence and variability of Si IV and C IV lines in HD 64740 spectra were used as proof of the circumstellar matter in the extended magnetosphere (Shore \& Brown 1990). However, hot Bp stars show an atmospheric component of Si IV and C IV lines (Kamp 1982), which may be variable if the elements are inhomogeneously horizontally distributed. Therefore, the circumstellar matter in the magnetosphere can be unambiguously detected only with detailed model atmospheres.

For silicon we have an abundance map at our disposal. Because the exact continuum placement is uncertain in the UV domain, Shore et al. (1987) devised certain indices to describe the line variability. Here we used similar indices defined using the flux at the line center (expressed in magnitudes $m_{\lambda}$ ) minus the average continuum flux,

$$
\begin{array}{r}
a(\mathrm{Si} \text { IV })=m_{1393.7}-\frac{1}{2}\left(m_{1388.6}+m_{1398.7}\right), \\
a(\mathrm{Si} \text { III })=m_{1205.9}-\frac{1}{2}\left(m_{1195.8}+m_{1226.0}\right), \\
a(\mathrm{Si} \text { III }+)=m_{1296.4}-\frac{1}{2}\left(m_{1289.7}+m_{1308.2}\right) .
\end{array}
$$

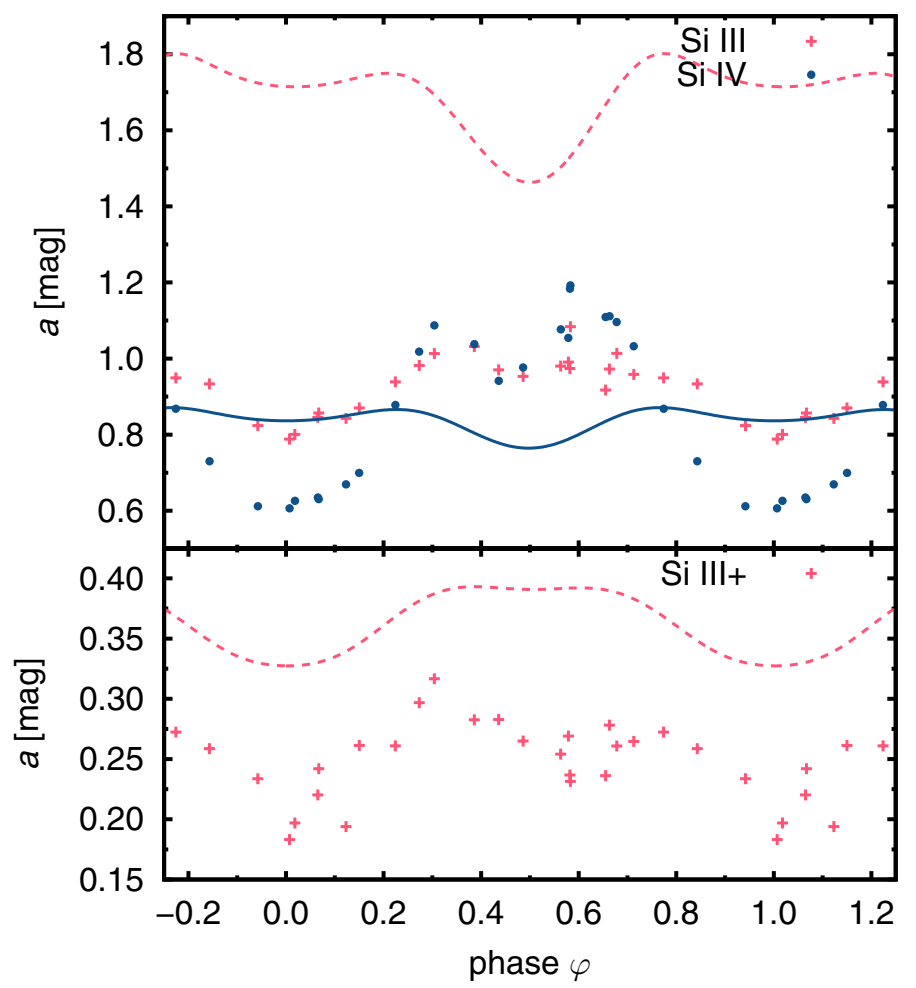

Fig. 6. Observed variability of silicon line indices Eq. (9) (Si III crosses, Si IV dots) compared with predicted ones (Si III dashed lines, Si IV solid lines).

We calculated the line indices from low-dispersion spectra. The wavelengths in Eq. (9) were selected from the spectra, and consequently do not exactly match the line centers. The comparison of the predicted and observed silicon line indices is given in Fig. 6. We detected the rotational line variability not only in Si IV lines as did Shore \& Brown (1990), but also in the resonance and excited lines of Si III (similarly to other Bp stars, Shore \& Adelman 1981). The variability in the resonance line of Si III at $1206 \AA$ has nearly the same amplitude as that of Si IV at $1394 \AA$ and $1403 \AA$. Observed and predicted line profiles disagree (Fig. 7). The predicted line profiles of Si III are significantly stronger than the observed ones, and the observed variability in the resonance lines has a larger amplitude than the predicted one. The observed Si IV line profiles (Fig. 7) are nicely reproduced only in phases $0.25-0.75$.

There are many strong carbon lines in the IUE spectra. Because we do not have any carbon abundance map at hand, we restrict ourselves to discussing their observed behaviour. We studied their variability using indices similar to those in Eq. (9)

$$
\begin{aligned}
& a(\text { C II } 1336 \AA)=m_{1335.0}-\frac{1}{2}\left(m_{1331.6}+m_{1340.0}\right), \\
& a(\text { C III } 1175 \AA)=m_{1175.7}-\frac{1}{2}\left(m_{1169.0}+m_{1180.8}\right), \\
& a(\text { C III } 1247 \AA)=m_{1247.8}-\frac{1}{2}\left(m_{1244.5}+m_{1249.5}\right), \\
& a(\text { C IV } 1548 \AA)=m_{1547.9}-\frac{1}{2}\left(m_{1529.4}+m_{1557.9}\right) .
\end{aligned}
$$

Many lines of different ionization states of carbon show strong line variability, including lines originating from excited levels (Fig. 8). The maximum of the carbon line strength occurs between phases $0.3-0.7$. On the other hand, we were unable 


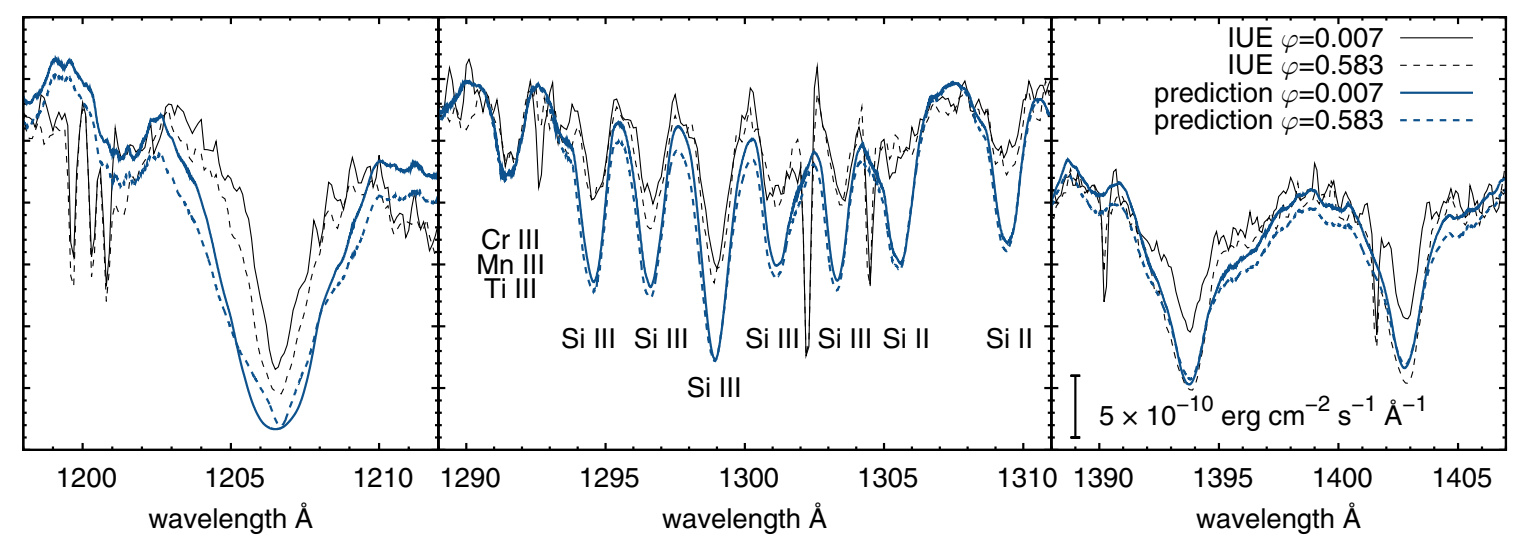

Fig. 7. Observed profiles of silicon lines (black) compared with predicted ones (blue) for phases $\varphi=0.583$ (solid lines) and $\varphi=0.007$ (dashed lines). Left: resonance line of Si III. Middle: excited lines of Si II and Si III. Right: resonance lines of Si IV.

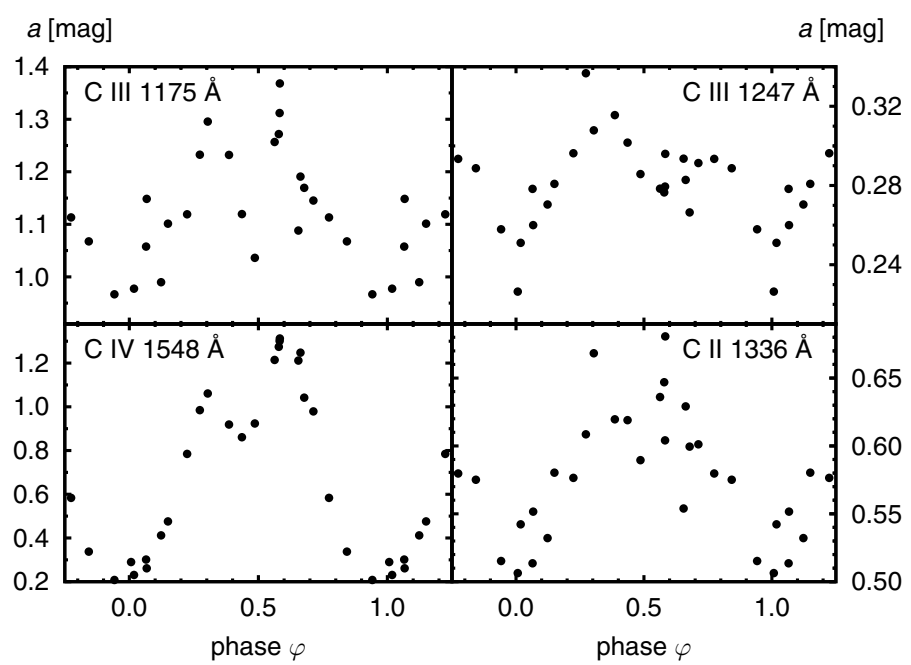

Fig. 8. Observed variability of carbon line indices according to Eq. (10).

to detect the rotational line variability of $\mathrm{C}$ II lines at $1721 \AA$ and $1761 \AA$, probably because of the weakness of the lines.

We also have a nitrogen abundance map available. However, the nitrogen lines are weak in the IUE range. Consequently, we were unable to detect any rotational line variability of the $\mathrm{N}$ II and $\mathrm{N}$ III lines. On the other hand, resonance $\mathrm{Al}$ III lines show distinct line variability with rotational period (as shown for other Bp stars by Shore \& Adelman 1981), as can be seen from the plot in Fig. 9 of the $1855 \AA$ line index defined as

$a(\mathrm{Al}$ III $)=m_{1854.7}-\frac{1}{2}\left(m_{1851.3}+m_{1858.0}\right)$.

Moreover, Al III resonance lines show the maximum strength around the phases $0.3-0.7$. Consequently, as can be seen seen in Figs. 6, 8, and 9, the variability of all the studied lines, expressed by means of indices (Eqs. (9)-(11)), show a similar behavior, regardless of whether the line is resonant or not.

The observed and predicted HST/STIS fluxes in the region 1920-2070 A agree well (see Fig. 10). All features in the spectrum are in fact blends of numerous lines, mostly of iron.

\section{Discussion}

\subsection{Photometric variability}

Three conditions have to be met so that appreciable photometric variability due to inhomogeneous elemental surface distribution

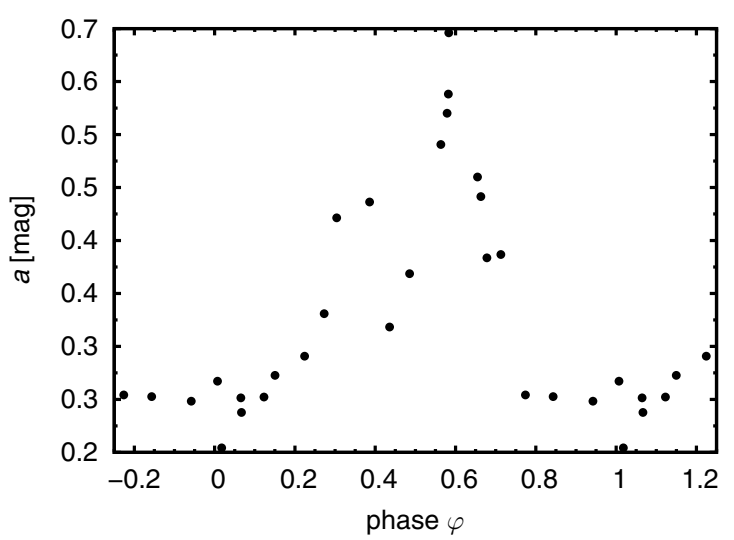

Fig. 9. Observed variability of the aluminium line index according to Eq. (11).

can be observed: i) an overabundance; ii) the amplitude of abundance variations of the photometrically active element have to be large enough to significantly influence the emergent flux; and iii) the surface spots should occupy a significant part of the stellar surface. The maximum overabundances of helium or silicon on the HD 64740 surface are not large enough to cause substantial flux redistribution. Consequently, although HD 64740 shows significant spectroscopic variations, its photometric variability in the UV and visual bands is hardly detectable. The light variations in stars where one of these conditions is not met have a very low amplitude and can be detected using spacebased instruments only (Balona 2011).

\subsection{Are co-rotating circumstellar clouds needed?}

Bp stars are well-known for their magnetically confined circumstellar clouds (Landstreet \& Borra 1978; Nakajima 1985; Smith \& Groote 2001; Townsend \& Owocki 2005). The matter lifted by the wind from the atmosphere flows along the magnetic field lines and accumulates in the local effective potential minima at individual field lines. The evidence for these corotating magnetically confined circumstellar clouds is particularly strong in stars with $\mathrm{H} \alpha$ emission (e.g., Townsend et al. 2005). The variability of UV lines is also frequently used as an indication of corotating clouds. However, we argue that this evidence may be problematic as a result of contamination of UV line profiles by atmospheric lines. 


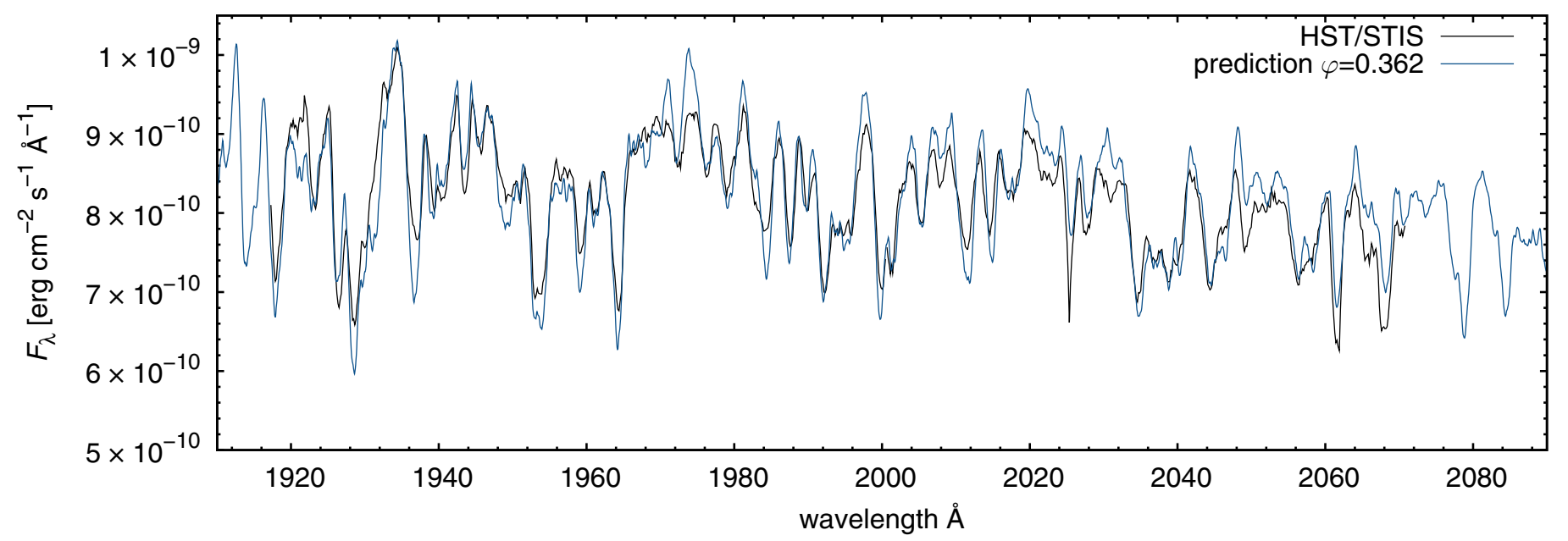

Fig. 10. Comparison of the predicted and observed narrow-range spectrum.

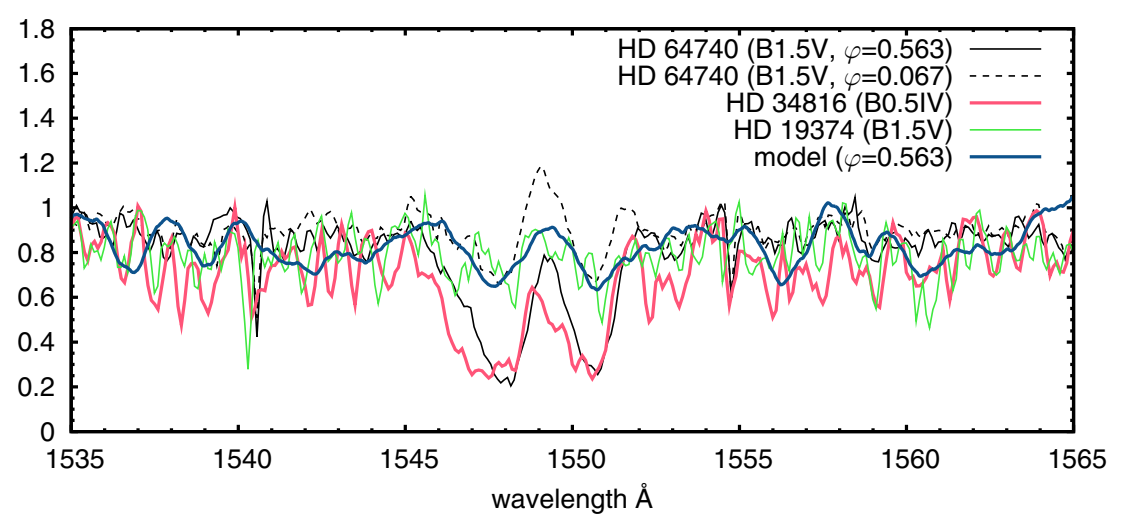

Fig. 11. Comparison of C IV resonance line profiles. The observed line profiles of HD 64740 are plotted in the phases of maximum and minimum line strengths. The predicted line profile was calculated assuming a homogeneous carbon abundance distribution, consequently it shows basically no variations. These line profiles are compared with IUE line profiles of normal stars with slightly earlier and the same spectral types than that of HD 64740. We note that the fluxes were normalized to a pseudo-continuum given by numerous absorption lines.

Detailed elemental abundance maps can be used to predict the UV line profiles of atmospheric origin and to test the nature of UV line variability. For HD 64740 the abundance maps are not sufficiently detailed, consequently, we had to use indirect tests to infer the origin of the UV line variability. The matter in the co-rotating magnetosphere accumulates in the warped disk with highest density above the intersections of magnetic and rotational equators (Townsend \& Owocki 2005). In HD 64740 this occurs for phases 0.25 and 0.75 . If the additional absorption occurs in the matter trapped in the magnetosphere, the strength of the CIV and Si IV lines should be strongest at these phases and there should be two roughly similar line strength minima at phases 0 and 0.5 . However, this is not the case; the line strengths increase at phase 0.25 and decrease at 0.75 (see Figs. 6 , 8, and 9). Moreover, the absorption lines should show profiles whose line center position slightly varies from blue to red, while the observed line profiles only show extended blue wings for the CIV lines (Fig. 11). Consequently, we conclude that it is questionable that the additional absorption occurs in the matter trapped in the rotating magnetosphere.

We note that although we found no clear signature of corotating magnetospheric clouds, HD 64740 most likely has such clouds. This is documented observationally from $\mathrm{H} \alpha$ line profiles (Alecian et al. 2012), and also theoretically, because the Kepler radius (at which the centrifugal force balances gravity) $R_{\mathrm{K}}=\left(\frac{G M}{\Omega^{2}}\right)^{1 / 3}=1.8 R_{*}$ is lower that the Alfvén radius, at which the wind speed is equal to the Alfvén velocity, $R_{\mathrm{A}} / R_{*}=$ $\left(\frac{B_{*}^{2} R_{*}^{2}}{\bar{M} v_{\infty}}\right)^{1 / 4}=28 R_{*}($ Owocki \& ud-Doula 2003). Here we assumed a wind mass-loss rate $\dot{M}=4 \times 10^{-10} M_{\odot}$ year $^{-1}$ and a wind terminal velocity $v_{\infty}=1700 \mathrm{~km} \mathrm{~s}^{-1}$, as predicted from NLTE wind models (Krtička \& Kubát 2010). The typical spindown time as a result of angular momentum loss via the magnetized stellar wind is about 6 Myr (ud-Doula et al. 2009), which can be measurable with a sufficiently long time span (Mikulášek et al. 2011).

We also tested if the accumulation of numerous iron lines might mimic absorption lines of C IV, as suggested by Hubený et al. (1986). For normal stars we confirm that the presence of absorption due to atmospheric C IV lines and numerous iron lines does not necessarily imply the existence of strong outflow, as shown in Fig. 11. However, these lines cannot explain the strength of absorption observed in HD 64740.

The strength and profile of C IV lines in HD 64740 does not correspond to its spectral type B1.5V, but to an earlier spectral type B0.5V (see Fig. 11 and Massa 1989). The extended blueshifted absorption in the spectra of early spectral types is the absorption part of the P Cygni profile originating in the stellar wind (e.g., Lamers \& Cassinelli 1999). Consequently, the strong C IV lines may originate in the stellar wind. In Bp stars several effects may strengthen the wind absorption lines. First, the wind mass-loss rate increases with metallicity (in $\mathrm{O}$ stars as $\dot{M} \sim Z^{0.67}$, Krtička 2006). Moreover, for a given mass-loss rate 

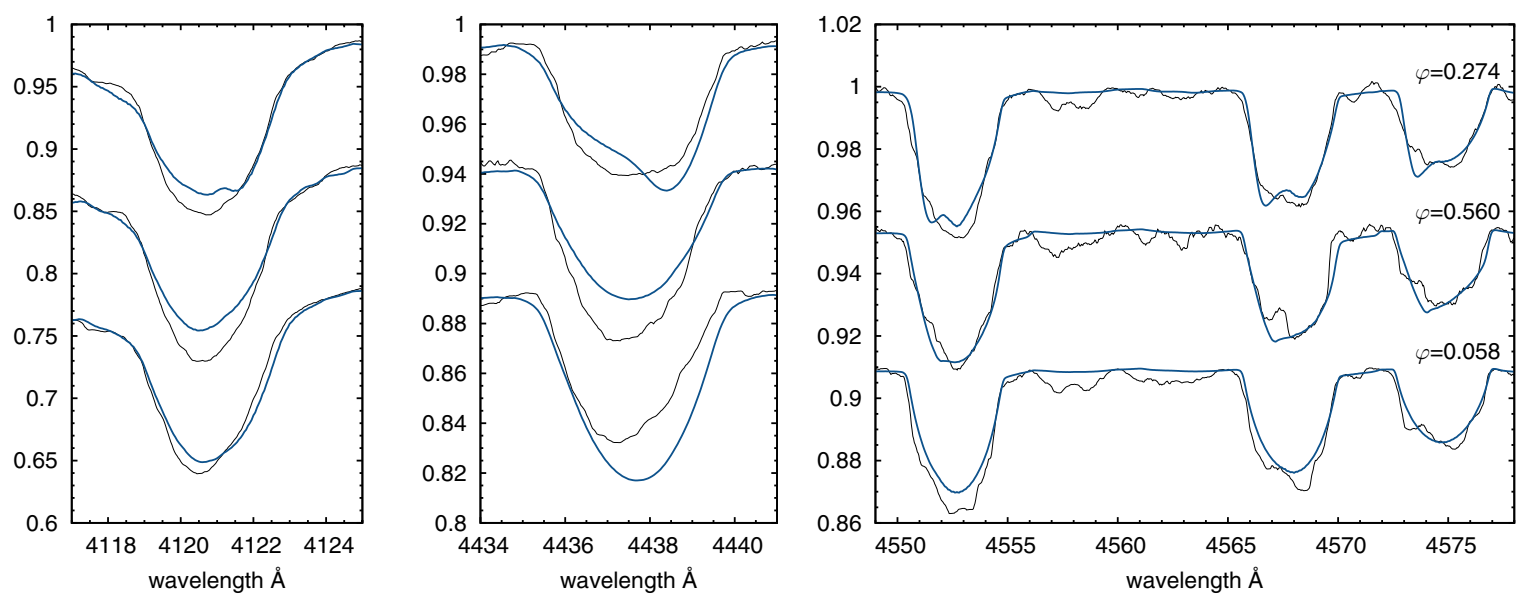

Fig. 12. Line profiles of individual helium and silicon lines in different phases (observed with FEROS) compared with line profiles predicted using abundance maps. Line profiles were vertically shifted to better demonstrate the line variability. Left and middle: Helium lines. Right: Silicon lines.

the line strength increases with increasing abundance of a given element. Mass-flux can be also affected by the geometry of the magnetic field (Owocki \& ud-Doula 2004). These effects combined may explain the strong C IV lines observed in the spectra of Bp stars, and with the inhomogeneous elemental surface distribution, also their variability.

From the variability of carbon line profiles in our FEROS optical spectra we infer that carbon is distributed inhomogeneously on the surface of HD 64740 . HD 64740 probably shows no enhanced carbon abundance (Lester 1976; Zboril \& North 1999). However, the strength of C IV resonance lines may reflect the enhanced mass-loss rate resulting from a higher abundance of other elements that drive the wind. This corresponds to the fact that regions with enhanced silicon abundance are visible in the phases $0.25-0.75$, where the carbon resonance lines are also the strongest. A similar effect may also affect the Si IV lines.

\subsection{Weak UV lines of Si II and Si III}

The predicted UV Si II and Si III line profiles are significantly stronger than the observed ones (Fig. 7). In Sect. 7.2 we argued that these lines and their variations probably do not originate in the circumstellar medium. There are other arguments in favor of an atmospheric origin of these lines. If these weak line profiles originate in the circumstellar medium, the photospheric absorption profiles have to be filled by the emission of circumstellar origin. However, the typical line profiles in such a case are different, showing emission in the wings in certain phases (Townsend et al. 2005). Moreover, resonance and excited lines are affected by the same relative amount, which is unlikely if the source of emission is the circumstellar medium.

The UV Si III line profiles can be better reproduced by models with an effective temperature higher by roughly $2000 \mathrm{~K}$. The published temperature determinations from the optical region seem to indicate the need for an opposite shift of the effective temperature (Zboril et al. 1997; Cidale et al. 2007; Netopil et al. 2008). However, because there is a relatively large uncertainty in the $T_{\text {eff }}$ determination (see Sect. 3.1 for a detailed discussion), the higher effective temperature can still be the reason for the disagreement between observed and predicted UV Si III line profiles.

Alternatively, a significant reduction of silicon abundance is required to bring the observations and theory in an agreement. The legitimacy of such an abundance reduction can in principle be tested using our optical FEROS spectra. However, as a result of the uncertainty of the adopted ephemeris we are unable to derive the reliable information about the phase for the FEROS observations. Consequently, if the abundances adopted by us are correct, the predicted and observed line profiles may differ in shape, but the line widths should be similar. This can be seen in Fig. 12. The abundance reduction therefore cannot explain the weak UV lines of silicon. We also note that the line profiles calculated by B88 from the maps agree with their observations.

The fact that the observed UV silicon lines that originate in the upper atmosphere are weaker than the predicted ones (while the remaining weak optical lines are not) may indicate a vertical silicon abundance gradient in the atmosphere (cf., Shavrina et al. 2010; Bailey \& Landstreet 2013). Our numerical test have shown that a significant reduction of the silicon abundance for the Rosseland optical depths $\tau_{\text {Ross }} \leq 0.01$ improves the agreement with UV line profiles of Si II and Si III while keeping the optical silicon line profiles nearly intact. The Si IV doublet at $1394 \AA$ and $1403 \AA$ is also to a lesser extent affected by the abundance stratification, but it is not clear if there is such a horizontal and vertical silicon distribution in the atmosphere that would fit all observations.

The adopted rather simple abundance maps are another possible source of the differences between theory and observations. The surface abundance maps are typically much more complex than those used here, and more detailed maps might possibly improve the agreement between theoretical and observed silicon line profiles.

\section{Conclusions}

We studied the UV and optical light variability of a Bp star with a very low amplitude of the light variability, HD 64740. We have shown that the maximum abundance of helium and silicon on the surface of HD 64740 is not high enough to cause a substantial light variability.

The observed and predicted UV flux distribution agree very well. The UV continuum is mostly given by numerous iron lines, and observations and theory agree particularly well in the region of the HST/STIS spectrum.

We detected the variability of numerous strong silicon UV lines and proposed that this is caused by abundance spots on the stellar surface. However, the overall agreement between predicted and observed line profiles is poor. Many UV silicon 
lines are much weaker than would correspond to the abundances deduced from the optical spectrum. The variable blueshifted resonance lines of C IV most likely originate in the stellar wind, whose mass loss rate (per unit of surface area) is spatially variable due to inhomogeneous elemental surface distribution. We found no clear evidence for the magnetically confined circumstellar clouds, whose presence can be detected (from the UV spectra) only when comparing the observed spectral line shapes with those predicted using detailed abundance maps of the corresponding element.

Acknowledgements. We wish to thank D. Bohlender for providing us his spectra and J. Kubát and M. Oksala for a discussion of this topic. This work was supported by the grant GA ČR P209/12/0217. This research was partly based on the IUE data derived from the INES database using the SPLAT package.

\section{References}

Adelman, S. J. 2001, A\&A, 367, 297

Alecian, E., Peralta, R., Oksala, M. E., \& Neiner, C. 2012, SF2A-2012, eds. S. Boissier, P. de Laverny, N. Nardetto, R. Samadi, D. Valls-Gabaud, \& H. Wozniak, 401

Asplund, M., Grevesse, N., \& Sauval, A. J. 2005, Cosmic Abundances as Records of Stellar Evolution and Nucleosynthesis, eds. T. G. Barnes III, \& F. N. Bash (San Francisco: ASP), ASP Conf. Ser., 336, 25

Bailey, J. D., \& Landstreet, J. D. 2013, A\&A, 551, A30

Balona, L. A. 2011, MNRAS, 415, 1691

Balona, L. A., Ripepi, V., Catanzaro, G., et al. 2011, MNRAS, 414, 792

Bohlender, D. A. 1988, Ph.D. Thesis, Univ. of Western Ontario (B88)

Bohlender, D. A., \& Landstreet, J. D. 1990, ApJ, 358, 274

Bohlender, D. A., Landstreet, J. D., Brown, D. N., \& Thompson, I. B. 1987, ApJ, 323, 325

Borra, E. F., \& Landstreet, J. D. 1979, ApJ, 228, 809

Briquet, M., Aerts, C., Lüftinger, T., et al. 2004, A\&A, 413, 273

Brown, D. N., Shore, S. N., \& Sonneborn, G. 1985, AJ, 90, 1354

Cidale, L. S., Arias, M. L., Torres, A. F., et al. 2007, A\&A, 468, 263

Drake, S. A., Linsky, J. L., Schmitt, J. H. M. M., \& Rosso, C. 1994, ApJ, 420, 387

Draper, P. W. 2004, SPLAT: A Spectral Analysis Tool (University of Durham), Starlink User Note, 243

Edgar, R. J., \& Savage, B. D. 1992, ApJ, 396, 124

ESA 1997, in The Hipparcos and Tycho Catalogues, Noordwijk, ESA SP, 1200

Hiltner, W. A., Garrison, R. F., \& Schild, R. E. 1969, ApJ, 157, 313

Harmanec, P., \& Horn, J. 1998, J. Astron. Data, 4, CD-ROM file 5

Harmanec, P., Horn, J., \& Juza, K. 1994, A\&AS, 104, 121

Hubený, I., Harmanec, P., \& Štefl, S. 1986, BAICz, 37, 370

Kamp, L. W. 1982, ApJS, 48, 415

Kochukhov, O., Wade, G. A., \& Shulyak, D. 2012, MNRAS, 421, 3004

Krivosheina, A. A., Ryabchikova, T. A., \& Khokhlova, V.L. 1980, Nauchnye Informatsii, Ser. Astrof., 43, 70

Krtička, J. 2006, MNRAS, 367, 1282

Krtička, J., \& Kubát, J. 2010, A\&A, 519, A50

Krtička, J., Mikulášek, Z., Zverko, J., \& Zižňovský, J. 2007, A\&A, 470, 1089

Krtička, J., Mikulášek, Z., Henry, G. W., et al. 2009, A\&A, 499, 567

Krtička, J., Mikulášek, Z., Lüftinger, T., et al. 2012, A\&A, 537, A14
Lamers, H. J. G. L. M., \& Cassinelli, J. P. 1999, Introduction to Stellar Winds (Cambridge: Cambridge Univ. Press)

Landstreet, J. D., \& Borra, E. F. 1978, ApJ, 224, L5

Landstreet, J. D., Silaj, J., Andretta, V., et al. 2008, A\&AL, 481, 465

Lanz, T., \& Hubeny, I. 2003, ApJS, 146, 417

Lanz, T., \& Hubeny, I. 2007, ApJS, 169, 83

Lanz, T., Artru, M.-C., Le Dourneuf, M., \& Hubeny, I. 1996, A\&A, 309, 218

Lehmann, H., Tkachenko, A., Fraga, L., Tsymbal, V., \& Mkrtichian, D. E. 2007, A\&A, 471, 941

Lester, J. B. 1976, ApJ, 210, 153

Lüftinger, T., Fröhlich, H.-E., Weiss, W. W., et al. 2010, A\&A, 509, A43

Massa, D. 1989, A\&A, 224, 131

Michaud, G. 2004, in The A-Star Puzzle, eds. J. Zverko, J. Žižňovský, S. J. Adelman, \& W. W. Weiss (Cambridge: Cambridge Univ. Press), IAU Symp., 224, 173

Mihalas, D. 1978, Stellar Atmospheres (San Francisco: Freeman \& Co.)

Mikulášek, Z., Krtička, J., Zverko, J., et al. 2007, in Physics of Magnetic Stars, Proc. International Conference, held in the Special Astrophysical Observatory of the Russian AS, August 28-31, 2006, eds. I. I. Romanyuk, \& D. O. Kudryavtsev, 300

Mikulášek, Z., Krtička, J., Henry, G. W., et al. 2011, A\&A, 534, L5

Molnar, M. R. 1973, ApJ, 179, 527

Moon, T., \& Dworetsky, M. M. 1985, MNRAS, 217, 782

Nakajima, R. 1985, Ap\&SS, 116, 285

Netopil, M., Paunzen, E., Maitzen, H. M., North, P., \& Hubrig, S. 2008, A\&A, 491, 545

Owocki, S., \& ud-Doula, A. 2003, in Magnetic Fields in O, B and A Stars: Origin and Connection to Pulsation, Rotation and Mass Loss, eds. L. A. Balona, H. F. Henrichs, \& R. Medupe (San Francisco: ASP), 350

Owocki, S. P., \& ud-Doula, A. 2004, ApJ, 600, 1004

Paunzen, E., Wraight, K. T.. Fossati, L., et al. 2013, MNRAS, 429, 119

Pedersen, H., \& Thomsen, B. 1977, A\&AS, 30, 11

Peterson, D. M. 1970, ApJ, 161, 685

Puls, J., Vink, J. S., \& Najarro, F. 2008, A\&ARv, 16, 209

Romanyuk, I. I. 2007, Astrophys. Bull., 62, 62

Ryabchikova, T., Kochukhov, O., \& Bagnulo, S. 2008, A\&A, 480, 811

Shavrina, A. V., Glagolevskij, Yu. V., Silvester, J., et al. 2010, MNRAS, 401 1882

Shulyak, D., Krtička, J., Mikulášek, Z., Kochukhov, O., \& Lüftinger, T. 2010a, 524, A66

Shulyak, D., Ryabchikova, T., Kildiyarova, R., \& Kochukhov, O. 2010b, A\&A, 520, A88

Shore, S. N., \& Adelman, S. J. 1981, in The Chemically Peculiar Stars of the Upper Main Sequence (Liège: Institut d'Astrophysique), 429

Shore, S. N., \& Brown, D. N. 1990, ApJ, 365, 665

Shore, S. N., Brown, D. N., \& Sonneborn, G. 1987, AJ, 94, 737

Škoda, P. 2008, Astronomical Spectroscopy and Virtual Observatory, eds. M. Guainazzi, \& P. Osuna, ESA, 97

Smalley, B., \& Dworetsky, M. M. 1995, A\&A, 293, 446

Smith, M. A., \& Groote, D. 2001, A\&A, 372, 208

Sokolov, N. A. 2006, MNRAS, 373, 666

Townsend, R. H. D., \& Owocki, S. P. 2005, MNRAS, 357, 251

Townsend, R. H. D., Owocki, S. P., \& Groote D. 2005, ApJ, 630, L81

ud-Doula, A., Owocki, S. P., \& Townsend, R. H. D. 2009, MNRAS, 392, 1022

Vauclair, S. 2003, Ap\&SS, 284, 205

Wamsteker, W., Skillen, I., Ponz, J. D., et al. 2000, Ap\&SS, 273, 155

Zboril, M., \& North, P. 1999, A\&A, 345, 244

Zboril, M., North, P., Glagolevskij, Yu. V., \& Betrix, F. 1997, A\&A, 324, 949 